\title{
Sagacious Communication Link Selection Mechanism for Underwater Wireless Sensors Network
}

\author{
Shahzad Ashraf ${ }^{* 1}$, Sehrish Saleem ${ }^{2}$, Tauqeer Ahmed ${ }^{1}$ \\ ${ }^{1}$ College of Internet of Things Engineering, Hohai University, Changzhou Jiangsu, China \\ ${ }^{2}$ Muhammad Nawaz Sharif University of Engineering \& Technology Multan Pakistan \\ Email:nfc.iet@hotmail.com
}

Received: 06 April 2020; Accepted: 13 May 2020; Published: 08 August 2020

\begin{abstract}
In underwater environment, the sensor nodes are deployed for collecting information and sending back to the base station. Establishing astute communication link among these sensor nodes in a multi-link routing environment is a key challenge for all underwater routing protocols. A sagacious communication link can only guarantee the maximum data transfer rate. The link selection mechanism of three underwater routing protocol i.e, Energy-aware Opportunistic Routing (EnOR) protocol, Shrewd Underwater Routing Synergy using Porous Energy Shell (SURS-PES) and Underwater Shrewd Packet Flooding Mechanism (USPF) have been investigated. After analyzing performance results of these protocols interms of packet delivery ratio, end-to-end delay, network lifespan and energy consumption using NS2 with AquaSim 2.0 simulator. The protocol existing, with sagacious link selection mechanism in multi-link routing environment has been identified. The identification of this sagacious link selection mechanism is a novel approach which can give specific knowledge for targeted output without wasting resources for irrelevant objectives.
\end{abstract}

Index Terms: Flooding mechanism, underwater routing, link selection, sagacious link, sink node

\section{Introduction}

Rummaging for oceanic resource, the underwater wireless sensor network plays a demanding role in this regard. It encompasses different varieties of sensor nodes according to the aquatic environment and meet the sensing data objectives. The deployed sensor nodes are fabricated with Radio Frequency (RF) and acoustic modem, used for sensing data related to atmosphere and unusual activities for instance, ocean and river pollution discovery, oil-andgas exploration, battlefield spying, building inspection, target-field imaging, disaster detection-and prevention, submarine-targeting, detection-of atmospheric-conditions such-as change-in temperature,-light, sound-or the-existence of-unlikely objects [1]. The sonobuoys (sinks) nodes float at upper water-surface and-responsible for-collecting related data-from these nodes. The-underwater sensor network is only feasible with acoustic channel. The acoustic signals operate at $1500-\mathrm{m} / \mathrm{s}$ which is less than the electromagnetic wave frequency in five order magnitudes [2]. The performance of the underwater network depends heavily on the topology architectural style, that is controlled by a selection mechanism for perfect embodiment deployed nodes which improves the probability of transmission to the destination node.

There are numerous challenges hindering to UWSN, the low bandwidth with absurdly high channel error rate, the transient route failure and weak channel multipaths, continue node displacement by $2-3-\mathrm{m} / \mathrm{s}$ at-water current [3]. Sound speed-is viewed stable-in the underwater-environment. However, sound intensity is influenced by the temperature, depth and salinity of the underwater ecosystem. These influences cause fluctuations in sound intensity in the underwater environment [4]. Underwater acoustic channel frequency range, particularly in mid-frequencies, is commonly shared by different acoustic users in underwater environments. Foremost, UWSN is facing an exorbitant energy wastage problem, all nodes are battery dependent and no mechanism has explored yet for replacement or charging. Therefore, in order to confine the energy wastage and utilizing the batteries potency efficiently, it is the only way to design a shrewd underwater routing protocols that should select the best and shorted rout between source and destination node [5].

The traditional terrestrial routing protocols are not well fitted in underwater environment. The optical and radio frequency interactions in such networks are generally deemed infeasible because optical signals suffer from extreme interference, while high-energy radio signals are easily absorbed due to high attenuation [6]. Establishing a communication link among deployed underwater nodes is major task of every underwater routing protocol and indeed it is a backbone of the routing mechanism. An underwater sensor network operates in multi-hop environment and thereby 
multi-routes are created from ground nodes to upper sink buoy [7]. It is crucial how and what metrics are considered for selecting best and stable link connection between these nodes because a stable communication link can ensure the data delivery operation and it is the only way that judge the performance of the network and overall throughput can be recorded. If the link becomes uncouth no smooth data packet delivery can be achieved, which results network failure [8].

The analysis of a sagacious link selection mechanism between sensor node under multi-routing environment is a main objective of this study. Therefore, meticulously research has been conducted in this regard and a communication link establishment mechanism is being analyzed for three state of the art underwater routing protocols i.e. EnOR, SURS - PES and USPF focusing on methodology by which each protocol works. In order to identify the best link selection mechanism by these protocols we have performed the quality test by conducting simulation using NS2 with Aquasim 2.0 simulator, in terms of packet delivery ratio, end-to-end delay, network lifespan and energy consumption. Finally, we identified the protocol and the sagacious link selection mechanism through which maximum performance can be achieved. We came across some challenges still faced by the underwater routing and that needs to be considered for future findings.

The underwater routing protocol controls the routing procedures through topology control mechanism. There are two types of routing topologies, i.e., Motion dependent, and Coverage dependent topologies [9]. The entire data routing and related issues are governed by these topologies. The salient aspects are highlighted as

\subsection{Motion based UWSN}

In order to monitor the other underwater component, sensor Nodes are located at a particular location such as the high water or low level. These distributed sensor nodes had different fidgets; floats, mostly operated by a navigation system, moving dynamically and continuously.

\subsection{Coverage based UWSN}

It consists mainly of the architecture of two dimensions and the architecture of three dimensions. For a twodimensional topology, the sensor nodes are anchored at the same depth and use the underwater communication bridge for the collection and transfer of raw data to base station. The sink nodes are equipped with a transceiver, which collects sensed data from the nodes that are above them. The typical sea level is estimated to be as much as $10 \mathrm{~km}$ and the vertical transceiver is to be sufficiently high, transmitting data to base station. Whereas the horizontal transceiver carries out the sensory node request for thrilling data acquisition. For multiple simultaneous communications, the sinks are mounted with an acoustic transceiver and a wireless transmitter. Underwater nodes may be linked directly to the sinknode from the in-line link through a multi-hop route (relay node). The direct link is a simple communication path but is not necessarily a resource-efficient choice for a sink node far from the sensor node. The power needed for transmitting packets may be decreased over far distance [10]. The network efficiency will also have high transmission capacities and significant acoustic interference could occur.

A multi-hop coordination technique raises the latency factor as data is interchanged in the maze of the routing. In addition, the two-dimensional underwater routing is confronted by main challenges in terms of communication ability; water surface selection and size. A 3D underwater sensor network is an advanced variant of 2D UWSN; the sensor nodes float freely to the arbitrary water depth, to capture sensed data. Sensor nodes are more jingoistic in their behaviour at the bottom and buoy holds the sensor nodes and it draws onto the water surface [11]. The anchor node depth can be controlled by adjusting the wire length. There are obstructed other obstacles to 3D underwater communication, including the sensor range must be naively modified in order to obtain sensory data intelligently and network topology associated.

The rest of the findings are structured as. Section II highlights the related findings. Section III addresses the characteristics of an acoustic signal. The link selection mechanism is discussed in section IV. Performance evaluation has been explained in section V. Some existing challenges are extensively highlighted in section VI, while conclusion and future research expectations are placed in section VII and VIII respectively.

\section{Literature Review}

The underwater sensor network has developed sagacious advancements in connection environment, media connectivity, mapping, and encoding and propagation technique. The energy use during the emergence of relay nodes is examined as an energy model in first exchange in [12], with sufficient energy route occupying exceptionally intermediate relay nodes at an optimal range, but without any implications for the forwarding structure. The author [13], suggested a vector-based forwarding mechanism (VBF), thereby a virtual pathway between source and destination node has been setup to deliver the data packets. The target node examines whether or not the packet is part of the virtual pipeline and, if so, validates factors that can be retained in time [14]. The packet is sent any time the same sender will quickly be forced to die, wasting exorbitant resources and growing the probability of packet loss. It is not possible for a fragmented network as pathways in virtual pipelines are fragile.

An energy-aware, void routing concept is blowing out by [15], (EAVARP), in which the author structures the conical shells around the sinking node and the sensor nodes inside such shells dynamically. Where data packets are inside the same shell, residual energy is transmitted that lets every empty area be circumvented if it occurs. However, 
the authors suggest an imaginative solution to the energy loss module, which as a result, shortens the lifetime of the network.

Huang et al [16], have designed a fuzzy-based routing approach to utilize the resource efficiently. The battery power is being utilized efficiently at a trivial level, the network overhead and packet delivery latency has been controlled. Indeed, in complex scenarios, this system increases uncertainty and offers no remedy for crash avoidance.

An esteemed QELAR method is proposed for the application of the adaptive routing protocol by Tiansi Hu et al [17]. It initiates a reward feature which uses the residual energy of sensor nodes evenly to be calculated and distributed, while nodes will remain alive throughout the application. The recompense function allocates relay nodes to the routing debut. Although the efficiency is awesome, but regular residual changes are bottlenecking in the network.

Sungryul et al [18], suggested a power transfer model making channel stability. The power effect has been evaluated by the amplitude of modifying waves, in the power transmission adjustment where the height of the wave determines the surface signal reflection and signal frequency transmitted to node. The value calculated may be used to determine the wave root mean square (RMS). Both nodes and corresponding frequencies should be equilibrated with wave height RMS, which is unable to be seen in the tests. The authors argue that the implementation of such a mechanism may lower significant energy consumption and thus reduce the transmission power of the node, but that no effective action to deal with this atrophy which can generate network congestion is addressed when the wave height is gradually reduced to empty level.

By dividing transmission to two parts, Cao, $\mathrm{j}$ et al [19], proposed the BTM balance transmission system, dividing the transmission into node results. Depending upon the energy level of the nearest nodes, nodes determine the singlehop or multi-hop spread of the data. The direct transference of one-hop data to the sink takes place if the voltage of the surrounding node is below the intensity level of the transmission node. Data is also transmitted to the destination sink through multi-hop transfers. This network integrates energy consumption in two opposing nodes. Nevertheless, there is only 1 relay node in each network, and unrealized transmissions are sometimes made when traffic, nodes and battery transmission effects are unevenly distributed.

\section{Salient Characteristic of Acoustic signal}

The communication in underwater ocean varies according to water level like deep and shallow water because of division of the oceans. The shallow water had high temperature, a multipath effect, a surface disturbance, and a broad pause in propagation that eventually adversely affected the output of sound signals; whereas a deep-water sea had the same history with specific measurements [20]. The acoustic signals when propagate in underwater environment faces numerous factors, therefore it is crucial to consider such underwater characterises before designing an underwater routing protocol. Some of these are highlighted as

\subsection{Factors affecting underwater Acoustic Communication}

In underwater environments, major propagating factors that affect acoustic communication are defined as:

i.) Path loss: indirectly the underwater temperature rises, which results in vacillated activity between sensor nodes and signal strength. The missing path has been further divided into three parts.

- Geometric Spanning Error: the sound wave is well removed from, but similar in wavelengths, as deep water induces spherical spreading damages and produces cylindrical absence of deep water stretching.

- Signal Attenuation: Attenuation is dependant on node frequency and the coverage [21]. That is because acoustic energy is transformed into another source of energy, namely heat energy.

- Dispersion: This occurs through shifting acoustic wave angles. Various speeds of wind cause surface ruggedness, which ultimately causes a delay and loss of power in subsequent communications to decrease the dispersion barrier.

ii.) High propagated delay: If the acoustic signal is $1500 \mathrm{~m} / \mathrm{s}$ the transmission is around $0.67 \mathrm{~s} / \mathrm{km}$ long and has a high propagation delay factor.

iii.) Noise Ratio: In order to add a noise ratio, the resulting signal amplitude state atrophies. Ambient noise [22], occurs in the case of UWSN attributable to several unidentifiable unknown causes. There exist four kinds of ambient noises as: (i) turbulence noise; (ii) shipping noise; (iii) wave formation noise, and (iv) thermal noise. Wind disruption generated by changing wind rates, allowing sound waves to break down. Acoustic transmission obstacles are induced by the acoustic wave distortion generated by the shipping journey. Sea surge produces low scale displacement, which creates disturbance during contact. In the course of communication. An automatic engine produces a sufficient noise, called an auto noise, which does not mimic other sounds. 
iv.) Multipath Enigma: The sounds waves affect the water surface and the depths of the oceans and create uncoherent acoustic communication interferences, which result in an inaccurate signal and a cross-cutting effect [23]. The waves are produced in underwater communication. The vector propagation and power effect dissidents are left by the impulse response of an acoustic system. Due to uneven speed of sounder, several paths are developed and the reflexion is only limited and energy loss is taken into consideration.

\section{Determination of Sagacious Link Selection mechanism}

The proposed topology of an UWSN with three different communication links has been illustrated in Fig.1. Deployed sensor nodes are broadcasting data packets towards neighbouring nodes within the transmission range thereby two transmission zones have been created. A successful created forwarding node transfers data to the sink node and then it is being transmitted to the offshore station for further processing. Communication links between source to relay nod and from relay node to sink node exhibits three different communication link status. A most stable link is being selected and thereby named sagacious link, while other links are ignored. A meticulous research regarding link selection mechanism from three state of art routing protocol have been investigated and performance is evaluated through which we concluded that which link selection technique gives maximum and shrewd performance by considering packet delivery ratio, end-to-end delay, network lifespan and energy utilization. The major findings regarding sagacious link selection mechanism of these protocols have been discussed one by one.

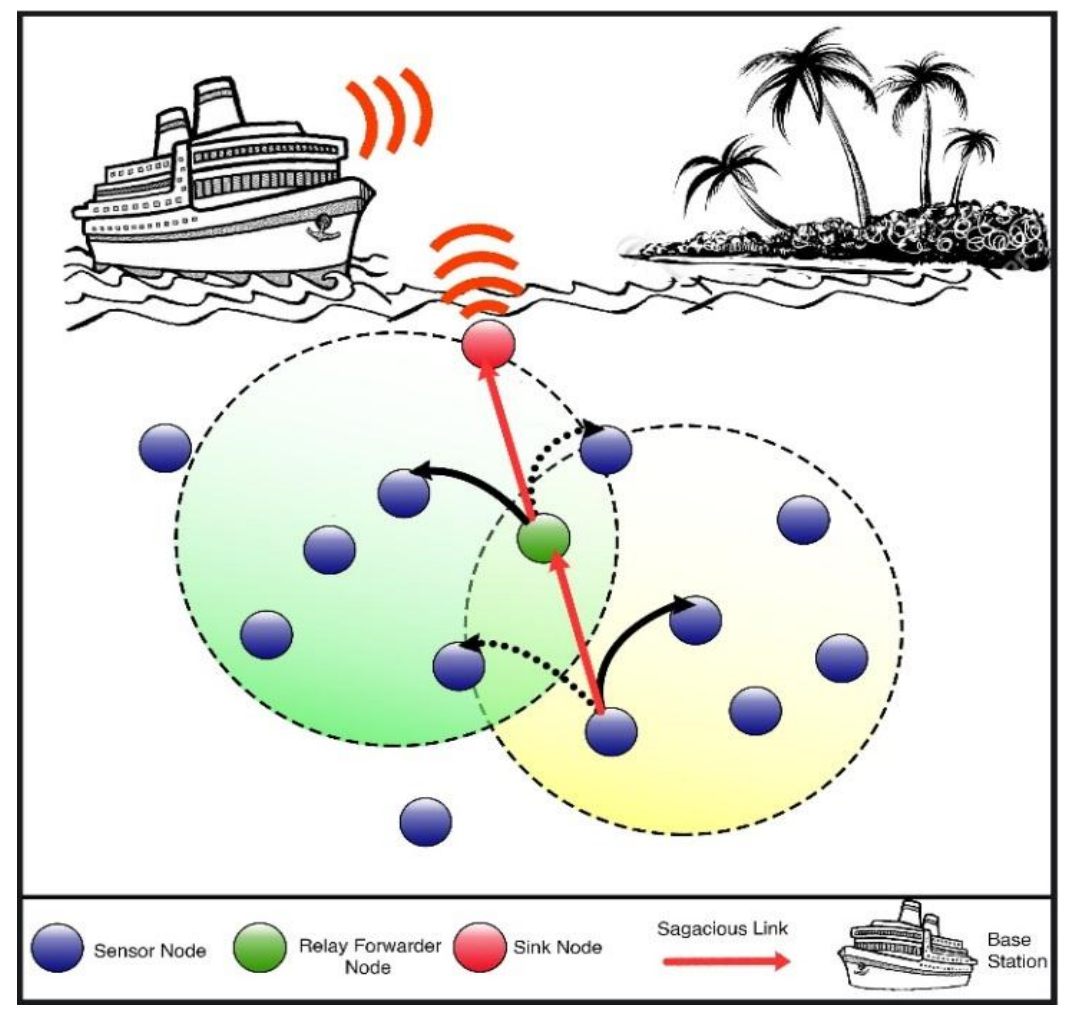

Fig.1. Underwater network topology presents three different communication links between source to destination (sink) node

\subsection{Energy Balancing Routing Protocol for Underwater Sensor Networks (EnOR)}

Rodolfo $W$ et al [24], addressed one of the immutability issues concerning the preference level of node transmission, resulted in optimized power consumption and prolonged UWSN network lifespan. Taking into consideration the remaining capacity, the efficiency of connections and packet development, it rotates the transmission priority level for transmission nodes.

Link Selection mechanism: Every underwater sensor node regularly transmits a beacon packet. The timing package consists of the sender's identity, and the remaining energy and size detail. The selection of sagacious links between source and destination node have been followed by the Algorithm 1. 


\section{Algorithm 1 explanation}

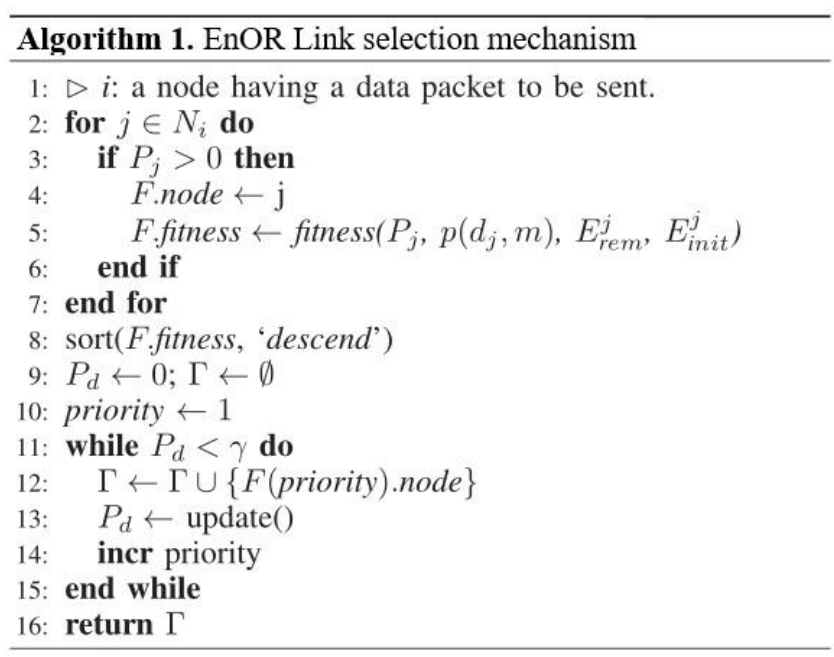

Considering $i$ being the source node carrying data packet and updates the record in neighbouring table Ni. The node analyzes its neighboring nodes and selects the most relevant transmitted nodes (lines 2-7). For such a feature, a candidate node can only be considered if a neighboring node progresses to a surface sonobuoy (sink). A difference between $i$ sender depth, and $j$ in $P_{j}=\operatorname{depth}(i)-\operatorname{depth}(j)$ is used to predict a packet forwarding for a neighbor $j$. The neighbor $j$ 's fitness is then determined (line 5). Using reliability of the connections to evaluate the suitability of a nearby node, packet advancement and energy remaining. The measurement is in line 5 and therefore as eq. (1):

$$
F_{j}=P_{j} \times p\left(d_{j}, m\right) \times\left(\frac{E_{r e m}^{j}}{E_{\text {init }}^{j}}\right)
$$

where $P_{j}>0$ shows packet forwarding of node $j ; p\left(d_{j}, m\right)$ presages of data packet $m$ from node $I$ to node $j$; $E_{j}$ rem shows remaining potency of $j$; and $E_{j_{\text {init }}}$ indicates starting metric of energy of $j$. The nodes have been used to select and sort the connection by fitness value (line 8). Eventually, the relation set is determined from the possible nodes. A restricted link collection will contribute to poor communication reliability. A wide connection set on the other side may also damage the query, which requires time. iThe potential node connectionsiare extended to the entire array until $\gamma$ is reached with the necessary link reliability.

\subsection{Design of Shrewd Underwater Routing Synergy Using Porous Energy Shells (SURS - PES)}

Authors [25], used a newly built relation to residual energy and depth information to relay the data packet from source to sink-node. The environment where energy consumption has a direct influence because all active underwater nodes depend on batteries and where cost-effective data packets are distributed, no charge or substitution measures would become a key factor. When a link becomes less than or equal to 50 percent weak, a shrewd link sorting procedure is used by the authors to search the destination node after the sensor node is transmitted, and the destination node returns to the source node, introducing an unusable porous energy shell to enrich the connection from 50 percent to 90.

Link Selection mechanism: The selection of a sagacious link inspection mechanism between sensor nodes is taken into account using the link reparation technique as shown in Fig.2. Sensor node, $p$, broadcasts the packet, $n$, with substantial information such as depth, ID, and residual energy towards neighboring nodes, i.e., q, $r$, and $s$. The source node, is broadcasting $p_{n}$ data packet towards neighbors, upon receiving this packet by the node $s$ includes necessary information and return this pack as $s_{m}$ to node $p$. When a duplicate node $p$ is attached to the required energy shells, the packet multizes again to node $\mathrm{s}$ as $2 p n$, in a trivial time $t$, The grain of the final relation is measured as shown in eq. (2). finally, the sagacious link has been chosen with sufficient energy consumption as $E p_{n}, E s_{m}$ and $2 E p_{n}$ accordingly.

$$
\text { Link Grain }=p_{n}+s_{m}+2 p n
$$




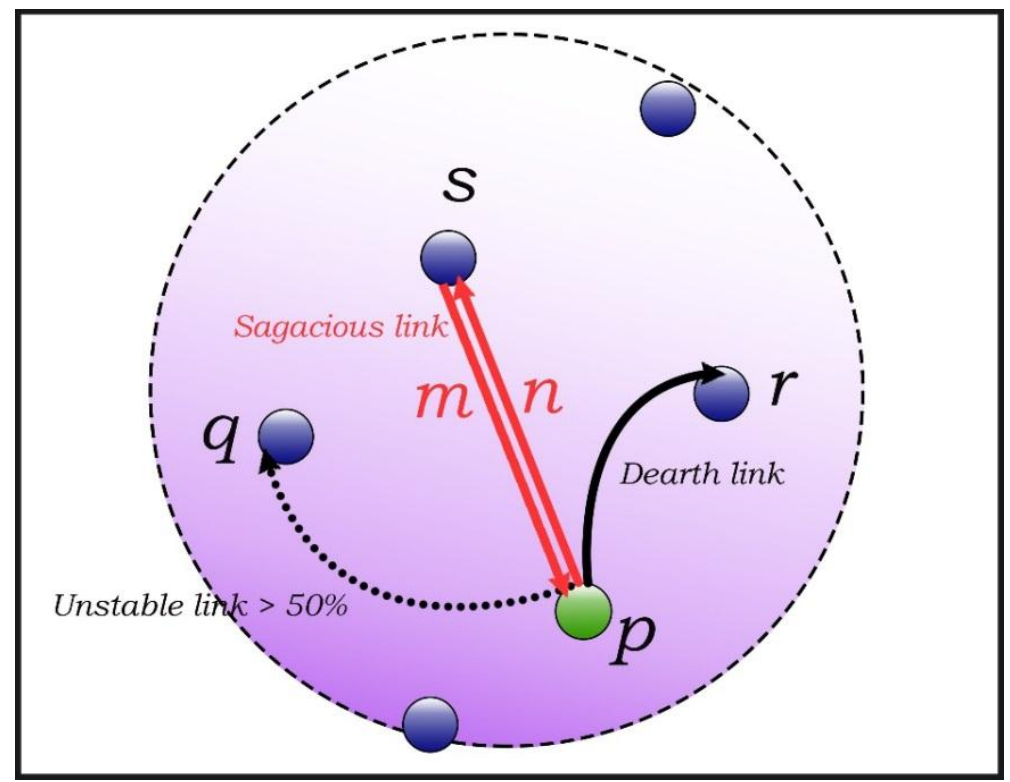

Fig.2. SURS - PES protocol represents a sagacious link selection mechanism

The energy utilization is by each participating node through every packet during transmission cycle is expressed as eq. (3), thereby link status updation can be predicted from 50\% to $90 \%$ in the due course cycle.

$$
\text { Link Grain }=t\left(\sum_{n=1}^{P_{n}} E p_{n}+\sum_{m=1}^{S_{m}} E s_{m}+\sum_{2 p n=1}^{2 p_{n}} E p_{2 p n}\right)
$$

There is a detailed analysis of the relations between node $p$ and others. There is also a mandated quality assurance link, which reports hop links that are more than 50 per cent affected and which links are more reliable than 50 percent at all. In relation to the stability of the link between the source node $p$ and $s$, the connection to the node $p$ and $q$ are more than 50 percent stable but not up to $90 \%$ indeed. The suggested solution (SURS-PES) therefore takes into account the hop link between node $p$ or $s$ for more stable packet transmission, i.e. up to $90 \%$.

\subsection{USPF: Underwater shrewd packet flooding mechanism through surrogate holding time}

An astute proposal was given by authors [26], for data packet transmission mechanism. Thereby three novel metrics where considered for link selection and pack holding time called surrogate holding time. The data packet broadcasts and network revitalization have been performed through an angle-based approach. All this accomplished in three phases. Forwarder Hop Angle (FHA) and Counterpart Hop Angle (CHA) are litigated to have data packets included in the first step of the same phase of transmission. If an FHA value is equal to or greater than CHA, the packet generated will be in the same transmission zone otherwise it would be claimed that the packet has other mavericks. The next move selects the best relay node again with a prefix-value accuracy using the Additive-Rise and Additive-Fall methods. At the end, the third phase provides a decisive solution to the exorbitant overhead fistula; the package holding period is designed to reduce the possibility of a packet failure.

Link Selection methodology: How shrewd is a link between source node $p$ and neighbouring node, is presaged by the Additive-Rise and Additive-Fall methods. Eventually it controls the link variations and make the link up to the acceptance level as depicted in Fig.3. 


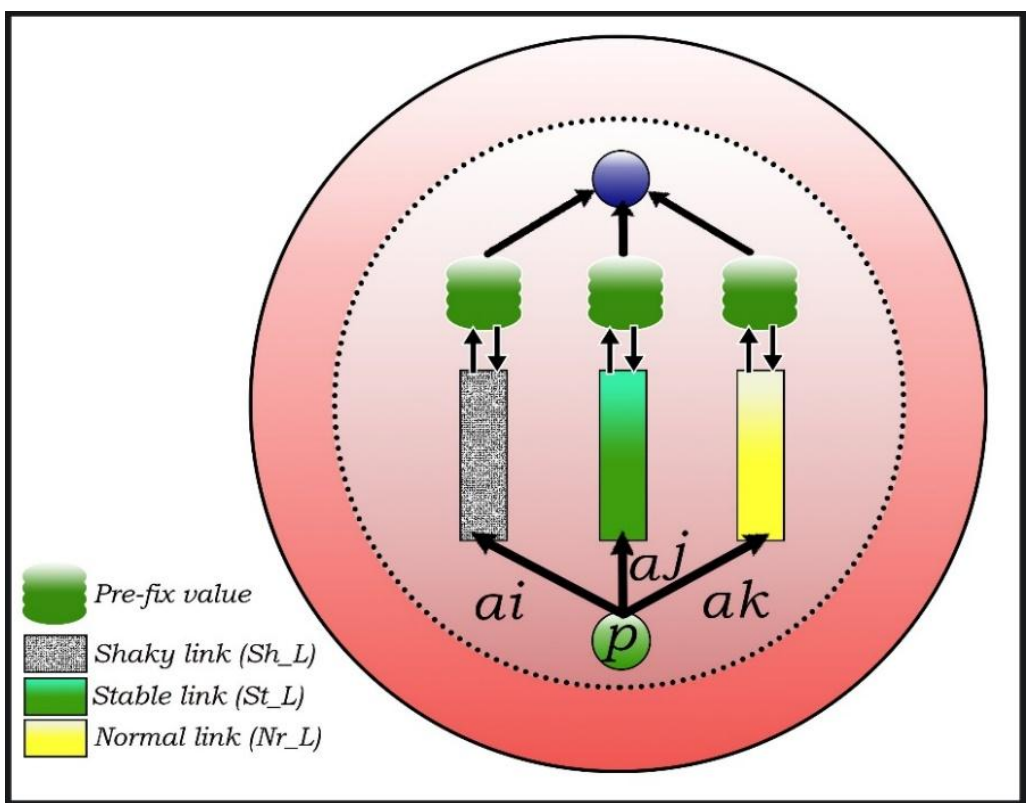

Fig.3. Using flooding mechanism, USPF protocol is selecting shrewd link connection

The description of aforementioned three phases are as follows:

Phase 1: Transmission node $\mathrm{p}$ switches the path to sluggish exploration when the connection state (Sh_L) becomes unstable or slanting as opposed to the prefix value (Prefix value) for the following nodes.

Phase 2: When the connection state (St_L) is stable and therefore the prefix (Prefix value) feature is fulfilled, the packet forwarding happens without obstacles.

Phase 3: When connection state $\left(\mathrm{Nr} \_\mathrm{L}\right)$ is normal, but not ready for transmission due to some salinity effects, other energy packets with additional shell, will go ahead. Measuring link quality from forwarding to neighboring nodes, thereby flooding zone is updated and metrics are expressed in eq. (4):

$$
F H A p=\left\{\begin{aligned}
\alpha i+s h_{-} L, & \text { if } \alpha i<\text { Prefix } x_{-} v \\
\alpha j, & \text { if } \alpha i=\text { Prefix } \_ \\
\alpha k+\left(N r_{L}\right)-\left(s h_{-} L\right), & \text { if } \alpha i>\text { Prefix_v }
\end{aligned}\right.
$$

The rising node updates the threshold value temporarily. The entire way of repairing the counterpart hop angle prevents the flood zone from being affected by the nullity, as the hop angle becomes complex metric in hop by hop fashion. Any relay node, however, is aware of counterpart hop angle in the nodes around it which appears to prevent nodes from engaging in the forwarding process.

\section{Performance Evaluation}

The link selection mechanism of three state of the art, underwater routing protocol has been meticulously investigated through a pragmatic approach in all thick and thins. To determine which protocol provides maximum performance by its link connection, the performance evaluation has been conducted through NS2 simulation setup with Aquasim 2.0. After examining the performance result, the sagacious connection is determined on the basis of the performance of the protocol i.e., EnOR, SURS - PES and USPF, interms of packet delivery ratio, end-to-end delay, network lifespan and energy consumption.

\subsection{Simulation setup}

About 300 sensor nodes have been deployed at different locations in 3D region with $800 \mathrm{~m} \times 800 \mathrm{~m} \times 800$ $\mathrm{m}$ dimensions while rest of the simulation parameters are given in Table.1. These simulation parameters are considered in accordance to the broadcast nature of opportunistic routing (OR) $[25,26]$ mechanism designed for underwater routing protocols. 
Table.1. Simulation setup parameters

\begin{tabular}{ll}
\hline Parameters & Setting \\
\hline Nodes quantity & 320 \\
Nodes initial energy & $48 \mathrm{~J}$ \\
Depth threshold & $8 \mathrm{~m}$ \\
Min: and Max: communication Range & $223 \mathrm{~m}, 245 \mathrm{~m}$ \\
Packet Size & $64 \mathrm{bytes}$ \\
Packet generation frequency & $0.03 \mathrm{pkts} / \mathrm{min}$ \\
Velocity & $1500 \mathrm{~m} / \mathrm{s}$ \\
Node displacement & $1 \mathrm{~m} / \mathrm{s}$ \\
Channel capacity & $10 \mathrm{Kbps}$ \\
Frequency channel & $24-28 \mathrm{kHz}$ \\
Transmission power & $1.8 \mathrm{~W} ; 0.75 \mathrm{~W} ; 8 \mathrm{~mW}$ \\
Data packet interval & $98 \mathrm{~s}$ \\
Channel bitrate & $140 \mathrm{~b} / \mathrm{s}$ \\
SNR for Signal Acquisition & $18 \mathrm{~dB}$ \\
Number of rounds taken for simulation & $500 \mathrm{rounds}$
\end{tabular}

\subsection{Packet delivery ratio}

The packets transmitted from the source node and reaching the final destination (sink) node are considered as packet delivery ratio. The average ratio of packet acknowledgment to the sink node of all protocols is analyzed in Fig.4. The delivery ratio is determined for EnOR, dependent on the percentage length reduction of the packet propagation, by a fraction of the obtained packets over the transmission process. This decline is due to the reduction in live nodes during the period. Comparing with SURS-PES, the shrewd packet turnout has increased with the number of nodes. This is because of scrupulous selection criteria for links and increased residual energy. Almost 100 to 250 nodes achieved an approximately $21 \%$ better smooth delivery ratio than EnOR. Finally, follow up USPF result, the improvements in the scale of flood zones instead of packet distribution culminated in unprecedented output witnessing $\mathrm{Nr} \_\mathrm{L}$ and $\mathrm{Sh} \_\mathrm{L}$ values, which gradually altered the $\mathrm{Sh} \_\mathrm{L}$ angle from 0 to $30^{\circ}$ and then $\mathrm{Nr} \_\mathrm{L} 0$ to $45^{\circ}$. Consequently, USPF brought unparalleled results in terms of packet distribution efficiency with other protocols.

\subsection{End-to-end delay}

With the rising number of nodes, the average end-to - end latency was stated to be decreasing for all protocols. In addition, this happens when the source is searching for qualifying nodes that can forward packets as seen in Fig.5., to the corroborated sink. This can be noted that EnOR also has a significant end-to-end latency relative to other EnOR networks because it has the largest difference in the transmitter and sink node owing to its priority fixation for rotary transmission relay nodes, in addition to a packet keeping length that varies on the priority nodes. While it worked well and achieved 200 nods when considering SURS-PES, the latency was significantly decreased although it stayed linear in other periods and this state remained similar in a dense and sparse environment. In view of USPF, the result is once again outstanding. According to its shrewd void prevention system, the USPF has achieved minimal end-to-end delays. The data from reception via transmission systems can be shown to a node in just 0.3 seconds so every relay is triggered for at least 0.3 seconds for every transmission cycle.

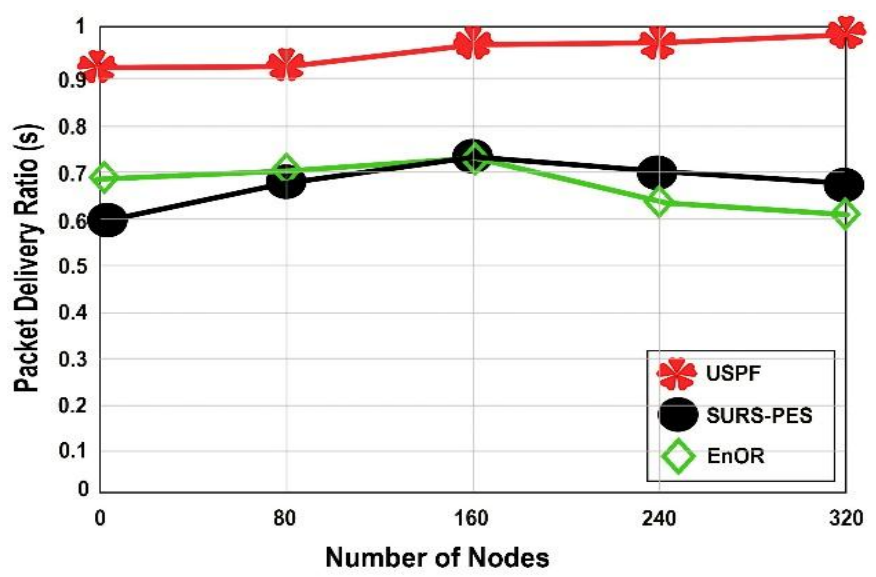

Fig.4. Average number of packets being delivered 


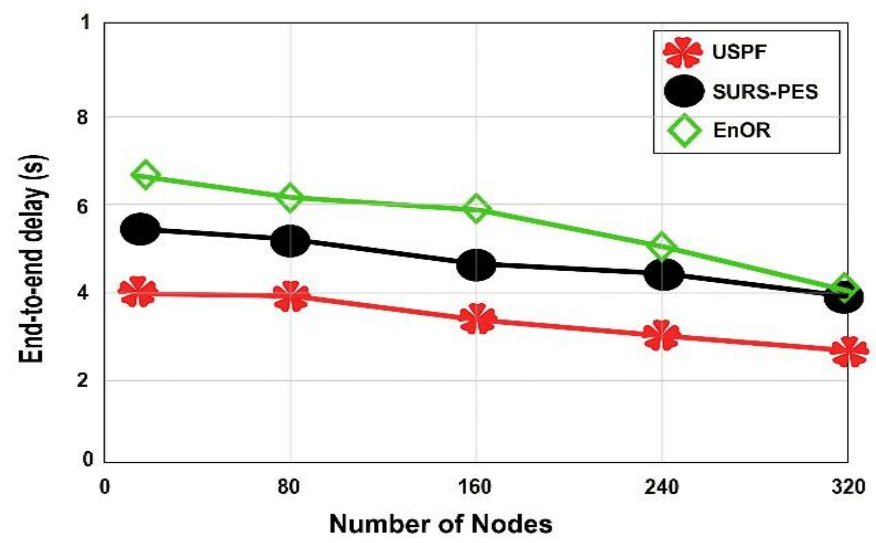

Fig.5. Amount of delay hiatus the packet to be ackknwledged

\subsection{Network Lifespan}

The cumulative lifetime is regarded as network life while nodes remain in the network. For EnOR, SURS-PES and USPF, the average network lifetime has been estimated, as shown in Fig.6. It should be remembered from EnOR that the precision of the node is quickly decreased by a large network density due to the energy costs involved with processing a packet and the nature of the propagation of the resulting acoustic communication that enables its reception from all its vicinity. Although SURS-PES protocol surpassed EnOR, it permitted the network's resilience to stretch during the transmission by the residual energy with shrewd link facilities. In fact, it has no packet keeping tangle, and no smooth packet motion is avoided irrespective of how high or limited the network bandwidth is. Network lifespan is therefore obviously less than that of USPF under the EnOR and SURS-PES protocols. It was considered because of various factors such as the residual energy when choosing the best forwarding node and the path selection mechanism with FHA and CHA adjust angles to prevent a void fistula.

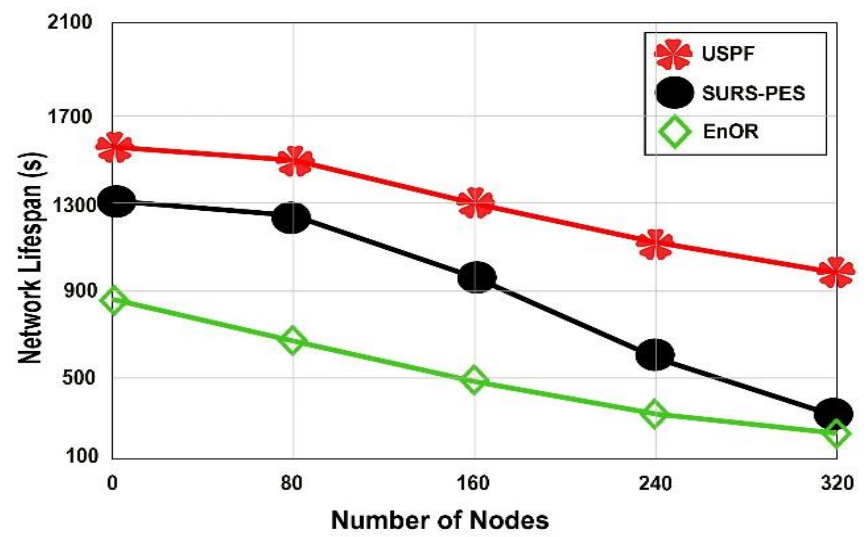

Fig.6. Total network alive time

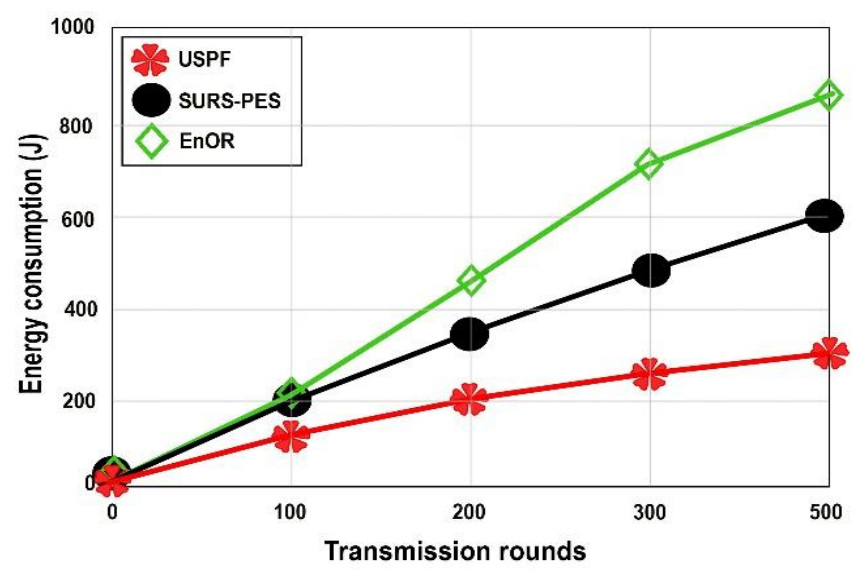

Fig.7. Amount of energy consumption by all nodes 


\subsection{Energy Consumption}

The total amount of energy required for the complete transfer of the packet from the source to the sink node is considered the energy usage of the network. The statistics shown in Fig.7., are provided in the simulation test. For each protocol USPF uses minimal resources for the total energy use of a number of nodes to reach a packet at the surface sink node since for each packet transmission the shrewd communication link is selected based on the additive rise and fall method and the time required for each relay node to keep the packet crashes and transmissions prevented. Although SURS-PES took into account residual energy and related efficiency for the use of restricted resources but cannot often use this technique, this requirement was initially faced by SURS-PES. The energy consumption by the nodes in the entire node network was slightly higher before the transition was produced. With regard to the EnOR Protocol the rotating priority level of transmission was used to absorb maximum energy to 200 nodes and performance was reduced.

\subsection{Results and Discussion}

Considering the cumulative efficiency of all protocols in terms of packet delivery ratio, end-to-end delay, network lifespan and energy consumption, it can be ratified that USPF has provided excellent results in all circumstances. The performance results are taken after 500 transmission rounds. For a packet delivery ratio, the sum of packets received by the sink node to the total sent was successfully received. This rises as the number of participating nodes has increased. It is worth noting that packet delivery ratio and the network lifespan are closely intertwined and directly proportional to each other. The result regarding network lifespan shows that in case of network congestion, still there exists reasonable adjustments, the packets may queue at the source and never reach the network. These packets do not attach to the output, but since they are never sent, they will not affect the packet delivery ratio at all. This directly affect the selection of sagacious communication link between source and destination node. According to the topology design perspective, all three underwater routing protocols have one common attribute; that is the packet delivery ratio, whereas for output point of view, the overall network lifespan is more important and often given higher priority than the packet delivery ratio. The link selection mechanism showed how significantly each protocol can genertate an opulent output. Comparing performance of all three protocols in termes of packet delivery ratio, end-to-end delay, network lifespan and energy consumption vouched that USPF has best link selection mechanism which gives maximum output in contracts to all rival protocols. Therefore the objective of this study has clrearly has ratified the statements which we made in introduction section.

\section{Existing Challenges}

The comprehensive findings regarding communication connection selection mechanism and its effect on different underwater routing factors were presented which revealed multiple obscure facets of this incredible area and several accessible problems still need to be tackled and left behind from which some are highlighted as.

- The structures of the noise and channel reduction are already in process for underwater routing. The theoretical or computational analysis and the development of new models are already possible in this area. Alternatively, concept versions may be created to reflect the features of the underwater medium.

- The sensor nodes are continuing, switching locations with water flows [19], thereby finding the sensor nodes is a daunting job. Also, node relocation requires regular adjustments in different locations. This creates uncertainty and the usage of resources, since such information will be exchanged at nodes.

- The protocols in this analysis require network layer into account. In order to increase network performance, the network layer can be combined with the MAC layer to reduce packet waiting in sensor node queues. This reduces disturbances when shipping packets, and consequently energy consumption. Intrusion continues to dissolve boxes, so damaged items are recovered and excess energy is consumed.

\section{Conclusion}

Finding sagacious link selection mechanism between source to destination node for three state of the art routing protocol was the key objective, which guaranteed the protocol to have a more significant long-term network live and optimum performance. Therefore, initially link selection mechanism was thoroughly investigated for EnOR, SURS-PES and USPF underwater routing protocols. Then in order to rummage which protocol possessed sagacious link selection mechanism, it was important to examine the performance of these protocols in terms packet delivery ratio, end-to-end delay, network lifespan and energy consumption. The performance results were obtained by conduction simulation through NS2 with Aquasim 2.0 simulator. The results showed that what is the affect of link selection mechanism on protocol performance and what happens if we change one parameter while keeping other same. The results ratified about EnOR protocol, that priority of spinning transfer is used up to the usable energy, stability and forwarding relay node advancement of the packet. At the process of SURS - PES, the packet is sent to the sink node from a source node, using a resurrect link metric with residual energy and depth data for choosing the best path. The USPF has introduced a 
newer strategy for link selection mechanism dependent on directional flooding, in view of two angles, that are Forwarder Hop Angle and Counterpart Hop Angle (CHA) with different packet keeping period. The overall results have vouched that across all transmission rounds, USPF remained best and worked extremely well in all stages therefore, it has sagacious link selection mechanism.

\section{Future Expectations}

There are open avenues to determine the impact of forwarding node impact on battery performance when packet transmission has been accomplished. The Packet Reverberation mechanism is still required in this area to work on it out, our next target is to expand our findings with this mechanism.

\section{References}

[1] S. Ashraf, M. Gao, Z. Chen, S. Kamran, and Z. Raza, "Efficient Node Monitoring Mechanism in WSN using Contikimac Protocol," Int. J. Adv. Comput. Sci. Appl., vol. 8, no. 11, 2017, doi: 10.14569/IJACSA.2017.081152.

[2] R. Diamant, P. Casari, F. Campagnaro, and M. Zorzi, "Leveraging the Near-Far Effect for Improved Spatial-Reuse Scheduling in Underwater Acoustic Networks," IEEE Trans. Wirel. Commun., vol. 16, no. 3, pp. 1480-1493, Mar. 2017, doi: 10.1109/TWC.2016.2646682.

[3] R. P. Hodges, Underwater acoustics: analysis, design and performance of sonar. Hoboken, N.J.: Wiley, 2013.

[4] A. S. Gupta and R. McCarthy, "Interpreting Different Features of Shallow Water Acoustic Channels Using Braid Manifolds," in 2018 Fourth Underwater Communications and Networking Conference (UComms), Lerici, Aug. 2018, pp. 1-5, doi: 10.1109/UComms.2018.8493234.

[5] D. A. Abraham, Underwater Acoustic Signal Processing: Modeling, Detection, and Estimation. Springer International Publishing, 2019.

[6] S. Ashraf, A. Raza, Z. Aslam, H. Naeem, and T. Ahmed, "Underwater Resurrection Routing Synergy using Astucious Energy Pods," J. Robot. Control JRC, vol. 1, no. 5, 2020, doi: 10.18196/jrc.1535.

[7] S. Ashraf, S. Saleem, and A. Zeeshan, "Diverging Mysterious in Green Supply Chain Management," Oriental J. Comput. Sci. Technology, vol. 12, no. 2.

[8] A. EL-Bakkouchi, A. Bouayad, and M. E. Bekkali, "A hop-by-hop Congestion Control Mechanisms in NDN Networks - A Survey," in 2019 7th Mediterranean Congress of Telecommunications (CMT), Fès, Morocco, Oct. 2019, pp. 1-4, doi: 10.1109/CMT.2019.8931405.

[9] M. Goetz and I. Nissen, "GUWMANET - Multicast Routing in Underwater Acoustic Networks," p. 18.

[10] K. Bruliński, A. Rudziński, and A. Stefani, "Characterization of ultrasonic communication channel in swimming pool," in 2019 Signal Processing Symposium (SPSympo), Sep. 2019, pp. 167-172, doi: 10.1109/SPS.2019.8882023.

[11] J. Liu, M. Yu, X. Wang, Y. Liu, X. Wei, and J. Cui, "RECRP: An Underwater Reliable Energy-Efficient Cross-Layer Routing Protocol," Sensors, vol. 18, no. 12, Nov. 2018, doi: 10.3390/s18124148.

[12] Q. JINGGUO, Z. ZILONG, and C. YUHUAN, "Research and Application of Multi-Node Communication and Energy Consumption Prediction Control in Underwater Acoustic Network," IEEE Access, vol. VOLUME 7, no. 2019, doi: 10.1109/access.2019.2907376.

[13] M. Safkhani, "Cryptanalysis of R2AP an Ultralightweight Authentication Protocol for RFID,” J. Electr. Comput. Eng. Innov., vol. 6, no. 1, Jan. 2018, doi: 10.22061/jecei.2018.1103.

[14] S. Ashraf, T. Ahmed, M. A. Khan, and Z. A. Arfeen, "Challenging strategic trends in green supply chain management," J. Res. Eng. Appl. Sci., vol. 5, no. 2.

[15] K. Guleria and A. K. Verma, "Comprehensive review for energy efficient hierarchical routing protocols on wireless sensor networks," Wirel. Netw., vol. 25, no. 3, pp. 1159-1183, Apr. 2019, doi: 10.1007/s11276-018-1696-1.

[16] H. Yang, Y. Zhou, Y.-H. Hu, B. Wang, and S.-Y. Kung, "Cross-Layer Design for Network Lifetime Maximization in Underwater Wireless Sensor Networks," in 2018 IEEE International Conference on Communications (ICC), Kansas City, MO, May 2018, pp. 1-6, doi: 10.1109/ICC.2018.8422176.

[17] R. W. L. Coutinho, A. Boukerche, L. F. M. Vieira, and A. A. F. Loureiro, "Performance modeling and analysis of voidhandling methodologies in underwater wireless sensor networks," Comput. Netw., vol. 126, pp. 1-14, Oct. 2017, doi: 10.1016/j.comnet.2017.06.027.

[18] R. M. Grant, "Prospering in Dynamically-Competitive Environments: Organizational Capability as Knowledge Integration," Organ. Sci., vol. 7, no. 4, pp. 375-387, Aug. 1996, doi: 10.1287/orsc.7.4.375.

[19] J. Cao, J. Dou, and S. Dong, "Balance Transmission Mechanism in Underwater Acoustic Sensor Networks," Int. J. Distrib. Sens. Netw., vol. 11, no. 3, p. 429340, Mar. 2015, doi: 10.1155/2015/429340.

[20] S. Balsamo, A. Marin, and E. Vicario, Eds., New Frontiers in Quantitative Methods in Informatics: 7th Workshop, InfQ 2017, Venice, Italy, December 4, 2017, Revised Selected Papers, 1st ed. 2018 edition. New York, NY: Springer, 2018.

[21] S. Das, A. Biswas, S. Dasgupta, and A. Abraham, "Bacterial Foraging Optimization Algorithm: Theoretical Foundations, Analysis, and Applications," in Foundations of Computational Intelligence Volume 3: Global Optimization, A. Abraham, A.E. Hassanien, P. Siarry, and A. Engelbrecht, Eds. Berlin, Heidelberg: Springer, 2009, pp. 23-55.

[22] C. Künzer and S. Dech, Eds., Thermal Infrared Remote Sensing: Sensors, Methods, Applications. Springer Netherlands, 2013.

[23] S. Ashraf et al., "Underwater Routing Protocols Analysis of Intrepid Link Selection Mechanism, Challenges and Strategies," Int. J. Sci. Res. Comput. Sci. Eng., vol. 8, no. 2, pp. 1-9, Apr. 2020, doi: 10.26438/ijsrcse/v8i2.19.

[24] R. W. L. Coutinho, A. Boukerche, L. F. M. Vieira, and A. A. F. Loureiro, "Geographic and Opportunistic Routing for Underwater Sensor Networks," IEEE Trans. Comput., vol. 65, no. 2, pp. 548-561, Feb. 2016, doi: 10.1109/TC.2015.2423677. 
[25] S. Ashraf, T. Ahmed, A. Raza, and H. Naeem, "Design of Shrewd Underwater Routing Synergy Using Porous Energy Shells," Smart Cities, vol. 3, no. 1, pp. 74-92, Feb. 2020, doi: 10.3390/smartcities3010005.

[26] S. Ashraf, M. Gao, Z. Mingchen, T. Ahmed, A. Raza, and H. Naeem, "USPF: Underwater Shrewd Packet Flooding Mechanism through Surrogate Holding Time," Wirel. Commun. Mob. Comput., vol. 2020, pp. 1-12, Mar. 2020, doi: $10.1155 / 2020 / 9625974$.

[27] T. Issariyakul and E. Hossain, Introduction to Network Simulator NS2, 2nd ed. Springer US, 2012.

[28] J. Qu, Z. Zhang, Y. Cui, J. Wang, and G. Mastorakis, "Research and Application of Multi-Node Communication and Energy Consumption Prediction Control in Underwater Acoustic Network," IEEE Access, vol. 7, pp. 41220-41229, 2019, doi: 10.1109/ACCESS.2019.2907376.

\section{Authors' Profiles}

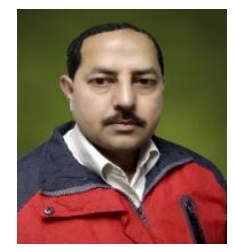

Shahzad Ashraf received B.E. degree in Computer Systems Engineering, and M.E. in Communication System and Networks from Mehran Engineering \& Technology University, Jamshoro Pakistan in 2004, and 2014 respectively. He is currently pursuing the Ph.D. degree in Information and Communication Engineering with the College of Internet of Things of Engineering, Hohai University Changzhou China. From 2005 to 2016, he served as an Assistant Professor at NFC Institute of Engineering and Technology Multan, Pakistan.

$\mathrm{He}$ is an active and prominent reviewer of many renowned international journals including IEEE, ACM, wireless personal communications, IET, IETE, international journal of distributed sensor networks, international journal of microwave and wireless technologies, journal of robotics and control, international journal of data science and analytics, international journal of computers \& technology, Technium: romanian journal of applied sciences and technology, iran journal of computer science, international arab journal of information technology, emerald: sensor review, international journal of pervasive computing and communications, journal of engineering and technology, and IGI global.

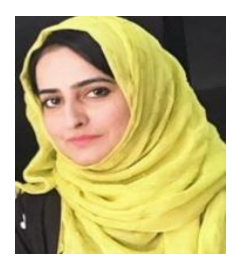

Sehrish Saleem did Bachelor of Science in Computer Science from NFC Institute of Engineering and Technology Multan and MPhil in Computer Science from National College of Business Administration \& Economics Lahore Pakistan in 2013 and 2016, respectively. She is serving as a Lecturer in Department of Computer Science at Muhammad Nawaz Sharif University of Engineering \& Technology Multan Pakistan. She focused on Software Engineering and her research interest includes Big Data Analytics, distributed Computing, Artificial Intelligence and machine learning.

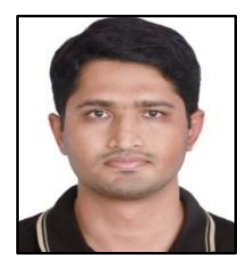

Tauqeer Ahmed received B.SC degree in Computer Systems Engineering and M.S in Electronic Engineering from International Islamic University, Islamabad Pakistan in 2011, and 2015 respectively. He is currently pursuing the Ph.D. degree in Information and Communication Engineering with the College of Internet of Things of Engineering, Hohai University Changzhou Campus China from 2018 to 2022, he served as an IT officer at Punjab Information Technology Board Multan, Pakistan. His research interests include Image processing, Signal processing, underwater acoustic sensor network, Computer hardware and networks.

How to cite this paper: Shahzad Ashraf, Sehrish Saleem, Tauqeer Ahmed, " Sagacious Communication Link Selection Mechanism for Underwater Wireless Sensors Network ", International Journal of Wireless and Microwave Technologies(IJWMT), Vol.10, No.4, pp. 22-33, 2020.DOI: 10.5815/ijwmt.2020.04.03 